\title{
Los trastornos de personalidad en reclusos como factor de distorsión del clima social de la prisión
}

\author{
JM Arroyo*, E Ortega***
}

*Médico Centro Penitenciario de Zaragoza

*Psiquiatra Centro de Salud de Rebolería Zaragoza. Pisiquiatra Consultor del Centro Penitenciario de Zaragoza

\section{RESUMEN}

Se estudia la prevalencia de los Trastornos de Personalidad (TP) en una prisión y su efecto en el clima social del establecimiento. El trabajo demuestra una relación estadísticamente significativa entre indicadores de desajuste del clima social en una prisión, como por ejemplo las conductas interpersonales agresivas o la demanda compulsiva de psicofármacos en las consultas médicas y el diagnóstico de TP. Este tipo de trastorno mental, uno de los más frecuentes en prisión, requiere especial atención por parte de los servicios sanitarios y su manejo es extraordinariamente complejo en el medio penitenciario.

Palabras clave: Trastornos de Personalidad, Prisiones, Relaciones Interpersonales.

\section{PERSONALITY DISORDERS AMONGST INMATES AS A DISTORTING FACTOR IN THE PRISON SOCIAL CLIMATE}

\begin{abstract}
The prevalence of Personality Disorders (PD) in a prison and its effect on prison social climate are studied. Our research shows a statistically significant relationship between diagnosis of PD and indicators of social climate disruption such as aggressive interpersonal conduct or compulsive demand for psychoactive drugs in medical consultations. PD is one of the most common mental disorders in prison, and requires special attention from health services, while management of the disorder is a tremendously complex issue in the prison context.
\end{abstract}

Key words: Personality Disorders, Prison, Interpersonal Relations.

Texto recibido: Septiembre 2008

Texto aceptado: Diciembre 2008

\section{INTRODUCCIÓN}

Una parte importante de las personas que ingresan en un centro penitenciario, lo hacen debido a un comportamiento socialmente inadaptado, en algunos casos como manifestación de un proceso patológico que les ha llevado a entrar en conflicto con la ley. En la prisión, se encontrarán en un ambiente caracterizado por el aislamiento afectivo, la vigilancia permanente, la falta de intimidad, la rutina, las frustraciones reiteradas y una nueva escala de valores que entre otras cosas, condiciona unas relaciones interper- sonales basadas en la desconfianza y la agresividad. Todos estos factores someten al recluso a una sobrecarga emocional que facilitará la aparición de desajustes en su conducta en el mejor de los casos, cuando no la manifestación de comportamientos francamente patológicos, sobre todo si previamente había ya una personalidad desequilibrada, en el momento de la entrada en prisión.

Coid en un extenso metaanálisis que recogía trabajos sobre morbilidad psiquiátrica en delincuentes ${ }^{1}$, concluyó ya en los años ochenta, que las prisiones tienen un nivel de morbilidad psiquiátrica mayor que el 
de la población general o incluso que el de la población antisocial no encarcelada, explorada antes de su ingreso en prisión en los Tribunales de Justicia. Mas recientemente en un estudio multicéntrico en nuestro país se demostró que las tasas de prevalencia de trastorno mental son muy superiores a las que habitualmente encontramos en la población general. ${ }^{2}$

Los trabajos que describen prevalencias de enfermedades mentales en prisión ${ }^{3-4}$, indican diferencias en patologías severas como las psicosis, entre la población penitenciaria y la población general, y un aumento importante en prisión de problemas adaptativos como los Trastornos de Personalidad, las toxicomanías y los Trastornos de Ansiedad, en comparación con las personas no encarceladas 5 .

La entrada en la cárcel pone en marcha un proceso de adaptación al entorno penitenciario, que muchos autores llaman prisonización y que se divide en tres niveles de afectación, el primero consiste básicamente en un comportamiento regresivo, inmaduro, ansioso e inestable desde el punto de vista afectivo como respuesta a la entrada en una Institución Total ${ }^{6}$ como es la cárcel. En caso de fallo adaptativo, un segundo estadio daría paso a verdaderos desórdenes de conducta, fundamentalmente marcados por comportamientos agresivos (auto o heteroagresividad), aparición de un deterioro afectivo depresivo o la presencia de episodios relacionados con trastornos de ansiedad en diferentes manifestaciones, bien somatizadoras, bien en forma de episodios ansiosos agudos. En un tercer nivel de este proceso de deterioro, aparecerá una patología mental severa, con brotes psicóticos, trastornos afectivos severos, reacciones vivenciales anormales o graves crisis de ansiedad e inadaptación a la prisión, lo que aconsejaría el ingreso hospitalario del recluso.

En este proceso de asimilación de la vida penitenciaria, es difícil repartir la influencia de los factores individuales y ambientales ${ }^{7}$, lo evidente es que cualquier mínima patología o disfunción que se presentara en el recluso o que éste padeciera antes de su encarcelamiento, sin un adecuado tratamiento, se agravará progresivamente mientras se mantenga al sujeto en ese medio, que desde el punto de vista del mantenimiento de la salud mental resulta tan exigente ${ }^{8-9}$. La presencia de un alto número de reclusos con patología psiquiátrica, no severa, pero sí conductualmente desadaptativa, tienen un efecto disfuncional sobre un clima social en la prisión debido a su falta de capacidad de ajuste al entorno, que transmite estrés a los propios internos y a los trabajadores del establecimiento que se relacionan con estos pacientes.

Este trabajo se ha centrado en el estudio de uno de los problemas de salud mental más prevalentes en los Centros Penitenciarios, los Trastornos de Personalidad (TP) proponíendolo como una de las causas de las relaciones interpersonales desadaptativas en un Establecimiento Penitenciario.

Los Trastornos de Personalidad, que pueden describirse como un estilo de relacionarse, de comportarse, de pensar y de afrontar dificultades, lo que es en definitiva una forma especial de personalidad patológica, suponen para los que los padecen una fuente de conflictos relacionales permanente ${ }^{10}$ ya que tienen como características centrales:

1. Poca estabilidad, tanto emocional como cognitiva, lo que conlleva rápidos y frecuentes cambios de humor e interpretaciones distorsionadas de la realidad

2. Inflexibilidad adaptativa en las relaciones interpersonales y con el entorno, con gran dificultad para reaccionar de manera modulada en función de las circunstancias, enfrentarse al estrés o reaccionar adecuadamente ante la frustración.

3. Tendencia a entrar en círculos viciosos o autodestructivos a consecuencia de las grandes limitaciones de su personalidad en las capacidades de adaptación al entorno.

En una institución cerrada, donde prima la seguridad, la restricción de movimientos y de espacios en los que realizarlos, el elevado control sobre los recluidos, el aislamiento, la ausencia de intimidad y la convivencia forzada entre sujetos, las relaciones interpersonales, frecuentemente cargadas de emotividad, son una de las principales fuentes de tensión para los reclusos y para los funcionarios con los que conviven. En condiciones normales, un sujeto que sufre una crisis vital que le hace cometer un delito grave, junto a la propia experiencia de la entrada en prisión, debe realizar un importante esfuerzo de adaptación para superar unas condiciones ambientales e individuales que pueden resultar tan adversas. Si en lugar de una personalidad equilibrada, nos encontramos con sujetos que presentan trastornos adaptativos previos, las dificultades de asimilación de la situación pueden ser insalvables. En estos casos la quiebra de la salud mental se traducirá frecuentemente en relaciones interpersonales patológicas.

La repetición de interacciones disfuncionales entre internos que presentan patologías adaptativas y trabajadores penitenciarios, puede incluir desde comportamientos agresivos, lábiles o incoherentes, hasta reacciones vivenciales francamente inapropiadas, la aparición de crisis de conversión o el desencadenamiento de una conducta psicótica. La sobrecarga 
emocional en este tipo de interacciones se vería notablemente disminuida si estos internos fueran correctamente diagnosticados y tratados, lo que contribuiría sin duda a mejorar un clima social de trabajo, siempre difícil ${ }^{11}$ y cuando menos, si los trabajadores penitenciarios fueran informados de la patología que presentan este tipo de internos y las reacciones que cabe esperar de ellos.

Debido a que la sintomatología de los Trastornos de Personalidad se manifiesta básicamente en el campo de las relaciones interpersonales y del comportamiento observable en diferentes circunstancias y ambientes, es frecuente que su diagnóstico resulte complejo $^{12-14}$. Ciertamente es necesario evaluar de manera continuada al mismo sujeto, para poder observar los comportamientos consistentemente inadaptados propios de este proceso. Por otra parte, los autores se dividen entre aquellos que consideran el Trastorno de Personalidad como un problema cuantitativo, en el que las dimensiones normales de la personalidad están alteradas por defecto o por exceso y otros que lo consideran un diagnóstico cualitativo, en el que determinados rasgos patológicos marcan la presencia del trastorno. En nuestra experiencia, este último modelo, defendido por la Sociedad Americana de Psiquiatría en su manual de clasificación DSM IV, es el que más eficientemente ha resuelto el diagnóstico y clasificación de estos pacientes ${ }^{15}$. La división de estos trastornos en tres grupos, A (paranoide, esquizoide y esquizotípico), B (antisocial, limite, histriónico y narcisita) y C (evitativo, por dependencia y obsesivocompulsivo), intenta clarificar la expresión polimórfica de estos cuadros. Otra dificultad añadida en el diagnóstico de estos procesos es la posibilidad de que aparezcan asociados entre sí o a otros cuadros psiquiátricos, cosa que ocurre frecuentemente en reclu$\operatorname{sos}^{16}$ donde es común la patología dual. En un estudio transversal hecho en prisión con una muestra de más de 2.000 internos, se ha descrito una prevalencia de adicción a drogas como la cocaína o la heroína en el $40,6 \%{ }^{17}$. De igual forma es frecuente la comorbilidad entre los reclusos, es decir la existencia de al menos dos trastornos mentales concurrentes en el mismo sujeto, hay diferentes trabajos que describen esta patología asociada en los TP ${ }^{18-19}$.

\section{MATERIAL Y MÉTODOS}

Este trabajo se ha realizado en un Centro Penitenciario de los llamados "tipo" con 14 módulos independientes, en el momento del estudio en el centro había 793 internos.
Utilizando un corte transversal, se obtuvo una muestra aleatoria de 60 internos en total, analizando dos grupos de 30 internos de módulos diferentes de la prisión. En uno de los módulos cumplían condena internos de los clasificados como mejor adaptados al régimen del centro y en el otro, internos de los clasificados como mal adaptados, debido a sus dificultades de convivencia y a su perfil criminal. Todos los internos eran varones.

Para medir el deterioro del clima social de la prisión, se utilizó un indicador referido a la interrelación de cada sujeto con el resto de sus compañeros y con los funcionarios en el módulo como puede ser el número de sanciones disciplinarias debidas a conductas agresivas. Otro indicador que se midió fue la demanda compulsiva de psicofármacos en la consulta médica, una actitud causante de estrés en personal sanitario por su relación con la frecuentación y los comportamientos agresivos durante el acto médico, ambos indicadores se recogieron durante 3 meses.

Se analizó la presencia de TP en las dos muestras de cada uno de los módulos.

Debido a la dificultad que existe en el diagnóstico, los casos considerados como TP en este trabajo, se consensuaron entre el Servicio Médico de la prisión, el Equipo de Psicólogos del Centro y el Psiquiatra consultor, eliminándose de la muestra aquellos que presentaban como primer diagnóstico: trastornos mentales orgánicos, oligofrenia, trastornos ansiosos, afectivos o psicóticos. La existencia de toxicomanía se recogió para ponerla en relación con el trastorno de personalidad. Se utilizó la versión española del cuestionario Examen Internacional de los Trastornos de Personalidad ${ }^{20}$ (IPDE), unido a entrevistas individuales no estructuradas que exploraban la sintomatología significativa para establecer los diagnósticos.

Se empleó la prueba de Chi-cuadrado para establecer la significación estadística de igualdad o desigualdad entre los valores de los indicadores de deterioro del clima social utilizados entre los sujetos diagnosticados de TP y los del grupo control.

\section{RESULTADOS}

Se observó un TP como primer diagnóstico en el $30 \%$ de los sujetos de nuestra muestra (18/60) que se distribuyeron según las siguientes frecuencias:

Trastorno Antisocial 11,6\% (7/60), Trastorno Límite $11,6 \%$ (7/60), Trastorno Paranoide 3,3\% (2/60), Trastorno Narcisista 1,6\% (1/60), Trastorno Esquizoide $1,6 \%$ (1/60) Predominan por tanto los TP del grupo B. 
Comparando a los sujetos del módulo de adaptados y a los del de inadaptados, observamos que había diferencias significativas en el número de toxicómanos encontrados, un 90\% (27/30) en el módulo de inadaptados y un $23,3 \%(7 / 30)$ en el de adaptados $(\mathrm{P}=0.0000007)$. Sin embargo no encontramos diferencias estadísticamente significativas en el número de Trastornos de Personalidad en ambos módulos, $(\mathrm{P}=0,398)$ ya que se diagnosticaron un $36,6 \%(11 / 30)$ de sujetos entre los clasificados como inadaptados en el centro y un $23,3 \%$ (7/30) entre los internos que se encontraban en el módulo de adaptados. Un 100\%, ya que todos los sujetos diagnosticados de Trastorno de Personalidad, eran también toxicómanos (Figura 1).

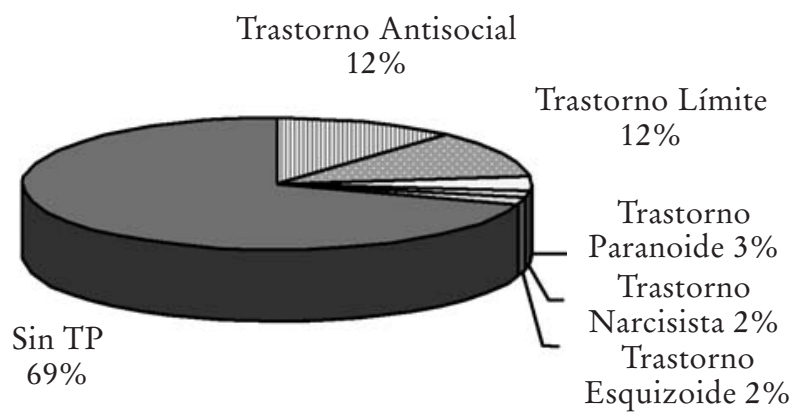

Figura 1. Distribución de TP.

Si relacionamos el número de sanciones disciplinarias debidas a actos agresivos y comportamientos que implicaban reacciones interpersonales inadecuadas con la presencia o no de Trastorno de Personalidad, un total de 11, observamos que el grupo de sujetos con estos trastornos presentaban el $90,9 \%$ de todas ellas (10/11), mientras el resto de sujetos tan solo el 9,09\% (1/11). ( $\mathrm{P}=0,007)$ los TP Limite el 50\% de todas ellas (5/10), los TP Antisocial el 40\% (4/10) y el 10\% el TP Narcisista (1/10) (Figura 2).

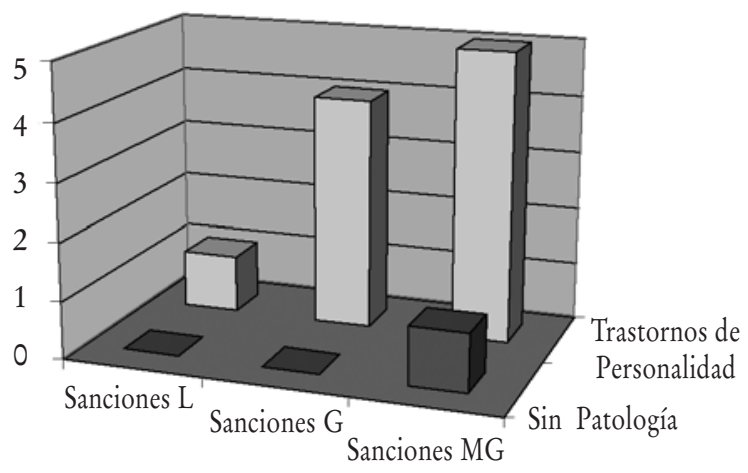

Figura 2. Sanciones y Trastornos de Personalidad.
Respecto a las demandas de psicofármacos, un $26,6 \%(16 / 60)$ sujetos de la muestra presentaban esta conducta, de los cuales el $75 \%(12 / 16)$ estaban diagnosticados de TP, una diferencia que resulta estadísticamente significativa $(P=0,04)$.

\section{DISCUSIÓN}

Uno de los factores que agrupa a la población penitenciaria de manera diferencial respecto a la no encarcelada, es precisamente su conducta ilegal. Parece lógico que como expresión de esta conducta ilegal, las personalidades con dificultades de adaptación tengan una presencia mayor entre los reclusos. Esto es explicable entre otras, por dos importantes razones, la primera porque es mucho más frecuente la historia previa de comportamientos inadaptados entre los delincuentes, antes de su entada en prisión ya que esta inadaptación está en la propia raíz del acto delictivo. La segunda, es que la estancia en prisión obliga a un esfuerzo permanente de ajuste y en este entorno, son puestas a prueba continuamente y muchas veces desbordadas, las capacidades de cada sujeto para la adaptación psico-social.

Hemos encontrado en nuestra muestra una prevalencia de TP acorde con la que otros trabajos describen $^{21}$. Esperábamos encontrar diferencias respecto al número de internos con TP entre el grupo de sujetos del módulo considerado inadaptado y el del considerado como de adaptados, sin embargo esto no ocurrió, probablemente porque entre las razones para clasificar a un interno en uno u otro módulo, no solamente se tiene en cuenta su perfil de personalidad, sino por ejemplo su pronóstico criminal, el tipo de delito cometido o el tiempo de condena que le resta. Estos factores no tienen porqué correlacionar con el grado de adaptabilidad del sujeto a su entorno social inmediato.

Hubo una relación estadísticamente significativa entre indicadores de desajuste del clima social en una prisión, como por ejemplo las conductas interpersonales agresivas o la demanda compulsiva de psicofármacos en las consultas médicas y el diagnóstico de TP. El número de internos con TP que se encuentra sin diagnosticar y sin tratar en las prisiones, progresivamente van empeorando en su sintomatología y con ello generan un deterioro cada vez más intenso en el clima social del establecimiento, que repercutirá tanto en el buen orden y la seguridad de la vida diaria en los módulos, como en el funcionamiento del equipo sanitario, que debe con frecuencia atender comportamientos incoherentes, impulsivos o francamente 
agresivos de estos pacientes. Nos parece especialmente importante el hecho de la alta presencia de patología dual en TP en prisión, lo que es un problema por el mal pronóstico de esta interacción ${ }^{22-23}$ y podría sugerir que el colectivo de toxicómanos en prisión, en una elevada proporción presenta a su vez trastornos de personalidad, lo que condicionaría su manejo.

La presencia del TP Límite en nuestra muestra es especialmente llamativa, ya que entra dentro de las esperadas en poblaciones que acuden a servicios psiquiátricos ${ }^{24}$, la de TP Antisocial es igualmente alta pero dentro de lo habitual entre reclusos.

\section{CORRESPONDENCIA}

josemarroyo@ono.com

\section{REFERENCIAS BIBLIOGRÁFICAS}

1. Coid J. "How many psychiatric patients in prison”. Br J Psychiatry 1984; 145: 78-86.

2. Hernández M, Herrrera R. La atención a la salud mental de la población reclusa. Madrid. Asociación Española de Neuropsiquiatría, 2003, 315-319.

3. Chelada C. More mentally ill people reported in US prisons. B M J, 1999; 24: 319-210.

4. Herrera R. Salud mental y prisiones. Rev Esp Sanid Penit. 2000; 2: 138-140.

5. Fanzel S, Dansen J. Serious mental disorder in 23000 prisoners: a systematic review of 62 surveys. The Lancet. 2002; 16: 545-550.

6. Clemente Díaz M, Los efectos psicológicos del encarcelamiento. En Jiménez Burillo F y Clemente M (eds) Psicología Social y Sistema Penal. Alianza Madrid, 1986: 253-268.

7. Hivert P. Psychiatrie en milieu carceral. Encycl Med Chir Psychiatrie 37889 A 9, Paris, 1982.

8. Tanay E. Psychiatry and the prison system. J Forensic Sci 1982; 27: 385-392.

9. Campos A, Gómez C. Efectos del internamiento en prisiones en la congruencia self-ideal. Rev Psiquiatr Psicol. Med Am Latina 1986; 17: 403-406.

10. Millon T Roger D D. Trastornos de la Personalidad. Más allá del DSM-IV. Barcelona. Masson. 1998: 3-17.

11. Caño M, Domínguez JM. Percepción del clima social en centros penitenciarios. Revista de Estudios Penitenciarios. Dirección General de Instituciones. 2000, No 248: 45-64. Dirección Gene- ral de Instituciones Penitenciarias Ministerio del Interior.

12. Ochoa E. Uso de antipsicóticos en drogodependientes. Actas Esp. Psiquiatr. 2001; 29: 172-185.

13. Iglesias García C, Gimeno Tejedor A, Díaz Conesa I, Inda Caro M. Evaluación dimensional de trastornos de personalidad definidos categorialmente, cinco factores vs. Clasificación DSM-IV. Estudio Piloto. Actas Esp. Psiquiatr 2000; 28: 71-76.

14. Svrakic D M. Personality Disorders. In Seva A. The European Handbook of Psichiatry and Mental Health II. Barcelona. Anthropos, 1991: p. 1763-1770.

15. DSM IV. Manual diagnóstico y estadístico de los trastornos mentales. Barcelona. Masson, 2001, p. 645-690.

16. Riesgo y Pérez Urdaniz A, Rubio V, Izquierdo JA, Sánchez Iglesias S, Santos JM et al. Evaluación de trastornos de la personalidad en una población penal con el IPDE y el MMPI. Actas Luso-Esp. Neurol. Psiquiatr. 1998; 26: 151-154.

17. Subdirección General de Sanidad Penitenciaria. Estudio Transversal sobre Sanidad Penitenciaria 22 junio 1998 Informe Interno. Ministerio del Interior, 1999.

18. Cervera Martínez G, Rubio Valladolid G, Haro Cortés G, Bolinches Claramontes F, De Vicente Manzano P, Valderrama Urian JC. La comorbilidad entre los trastornos del control de los impulsos, los relacionados con el uso de sustancias y los de la personalidad. Trastornos Adictivos 2001; 3: 3-13.

19. Ochoa Mangado E. Cocaína y comorbilidad psiquiátrica. Actas Esp Psiquiatr 2000; 28: 40-52.

20. OMS. Loper Ibor J J Examen Internacional de los Trastornos de la Personalidad (IPDE). Módulo DSMIV. Madrid. Meditor, 1996.

21. Pérez Urdaniz A, Santos García JM, Molina Ramos R, Rubio Larrosa V, Carrasco Perera JL, Mosquera Terron I, et al. Aspectos socioculturales en la génesis de los trastornos de personalidad. Actas Esp Psiquiatr 2001; 29: 47-57.

22. Pérez de los Cobos J. Tratamiento farmacológico de los trastornos y cambios de personalidad concomitantes a una adicción. Trastornos Adictivos, 2001; 3: 25-32.

23. Sánchez Hervas E. Trastornos adictivos y otros trastornos mentales. Psiquiatría Biológica 2001; 5: 61-73.

24. Svrakic D M. Personality Disorders. In Seva A. The European Handbook of Psichiatry and Mental Health II. Barcelona. Anthropos 1991: p. 1766-1767. 